\title{
Viewpoint: Celebrating Medical Librarians During Canadian Library Month
}

\section{Danielle Rabb}

\section{Key Messages}

- October is Canadian Library Month and an opportunity to acknowledge the work done by medical librarians in response to the COVID-19 pandemic.

- Librarians serving both the research community and the public were quick to adapt to the restrictions imposed by COVID-19. They provided us with access to electronic books, journals, and article databases and helped us via live chats.

- Curating information about COVID-19 has involved partnerships between CADTH, Canadian research libraries, and overseas organizations.

The fast-moving and extensive nature of the COVID-19 pandemic and the sudden need for a credible research base to support those trying to understand the coronavirus have shone a spotlight on the key role librarians play in finding and providing access to reliable medical information.

With October designated as Canadian Library Month, and COVID-19 still very much with us, it seems an appropriate time to acknowledge the essential role of librarians during the pandemic.

Medical librarians have always offered the service of making sense of and organizing emerging data and evidence, and this has never been more important than during the pandemic. The medical librarians who support the research community were quick to adapt to the restrictions imposed by COVID-19 and to meet the needs of the people who rely on their services.

Library services have been steadily providing more online services, but the pandemic required libraries supporting the academic community to pivot and offer everything online from access to electronic books, journals, and article databases to providing live chat help. To do so required meeting unique technological, licensing, and human resource challenges.

Curating information about COVID-19 also become an important function. The Canadian Association of Research Libraries (CARL), which represents 29 of the larger Canadian university libraries and 2 federal government libraries, collaborated with OpenAIRE - a discovery service funded by the European Commission - to develop a special search portal that provides access to all research outputs related to COVID-19. 
At CADTH, the Research Information Services (RIS) team quickly rallied at the onset of the pandemic to monitor and keep track of key resources related to the emerging evidence base. As the number of sources of COVID-19 information grew, the RIS team compiled these sources into a COVID-19 Grey Literature Resources list, which was published on the CADTH COVID-19 microsite. The team also developed a unique set of key database filters for COVID-19-related topics: CADTH COVID-19 Search Strings.

The CADTH RIS team was also invited to join the Librarian Reserve Corps (LRC), a WHO Global Outbreak Alert and Response Network partner. With the LRC, the RIS team undertook a validation study of several specialized COVID-19 databases that were developed in response to the pandemic to evaluate their completeness and currency.

The nimbleness of the library community in shifting to meet changing needs during the pandemic is not confined to the world of academia - public libraries also evolved their operations to meet changing needs during the pandemic while investing in more online and virtual content and providing new digital tools.

Medical librarians across Canada and around the world play an essential role in scientific research and knowledge dissemination. Their skills in critical appraisal, knowledge management, and advanced search methodologies are vital across the stages of health technology assessment, and their well-established role within interprofessional research teams will only continue to expand post-pandemic. 